\title{
Mechanical properties and finite element analysis of walnut under different cracking parts
}

\author{
Hong Zhang ${ }^{1}$, Liuyang Shen ${ }^{1,2^{*}}$, Haipeng Lan ${ }^{1}$, Yong Li ${ }^{1}$, Yang Liu ${ }^{1}$, Yurong Tang ${ }^{1}$, Wen $\mathrm{Li}^{1}$ \\ (1. College of Mechanical and Electronic Engineering, Tarim University, Alar 843300, China; \\ 2. College of Engineering, Northeast Agricultural University, Harbin 150030, China)
}

\begin{abstract}
In order to reduce mechanical damage and improve extraction quality of walnut kernel during walnut cracking, the Wen-185 walnut cultivar was selected as the research object, and the mechanical properties of walnut under different cracking parts were assessed by combining compression tests and finite element analysis (FEA) method. The compression test results showed that the relationships between rupture force and deformation of walnut were nonlinear, and the cracking process of shell mainly consisted of three stages (elastic stage, plastic stage and composite elastic-plastic stage). The best method to crack walnut was the spherical compression, and the peak value of rupture force and corresponding deformation were $211.83 \mathrm{~N}$ and $1.68 \mathrm{~mm}$, respectively. In condition of spherical compression, the shell-breaking rate, first-grade kernel rate and whole kernel rate were $(91.67 \pm 2.89) \%,(88.33 \pm 2.89) \%,(80.00 \pm 5.00) \%$, respectively. The FEA results indicated that spherical compression was also the suitable way to rupture walnut, which resulted in the obvious propagation trends of shell cracks and further a better integrity of extracting walnut kernel. Therefore, the spherical contact form between walnut and cracking parts may be considered to design the structural shape of key components of walnut cracking machines, which was consistent with the analysis of compression test results. The comparison between experiment results and FEA results showed that the established FEA model can be used to analyze the mechanical properties of walnut. The research results can provide references for the structural design and optimization of key components of cracking machines for walnut or other nut crops.
\end{abstract}

Keywords: walnut, mechanical damage, compression tests, mechanical properties, finite element analysis DOI: $10.25165 /$ j.ijabe.20181106.3309

Citation: Zhang H, Shen L Y, Lan H P, Li Y, Liu Y, Tang Y R, et al. Mechanical properties and finite element analysis of walnut under different cracking parts. Int J Agric \& Biol Eng, 2018; 11(6): 81-88.

\section{Introduction}

Walnut (Juglans regia L.) is an excellent source of highly nutritional components such as fatty acids, proteins, vitamins and a variety of minerals, and has been reported to decrease the risk of coronary heart disease, which is considered one of the most nutritional nuts and deeply loved by consumers ${ }^{[1]}$. China is ranked first in the world with $1602373 \mathrm{t}$ of walnut production ${ }^{[2]}$. Xinjiang Uygur Autonomous Region, which has a long history of walnut planting, is the second largest production base of walnut in China, but over $90 \%$ of walnut are cultivated in the southern area of Xinjiang (hereinafter referred to as South Xinjiang) ${ }^{[3]}$. The cracking process of walnut is the most critical and delicate step for achieving high-quality kernels, yet walnut cracking is until now carried out manually in South Xinjiang, which leads to increased

Received date: 2017-05-26 Accepted date: 2018-04-09

Biographies: Hong Zhang, Master, Professor, research interests: agricultural machinery and equipment design, Email: zhghog@163.com; Haipeng Lan, PhD, Associate Professor, research interests: agricultural products processing technology, Email: lanhaipeng@126.com; Yong Li, Master, Associate Professor, research interests: agricultural machinery and equipment design, Email: deyuzhijia@163.com; Yang Liu, Master, Lecturer, research interests: agricultural products processing technology, Email: hxtxylove@126.com; Yurong Tang, Master, Laboratory Technician, research interests: agricultural mechanization and automation, Email: tangyurong026@163.com; Wen Li, Master candidate, research interests: agricultural machinery and equipment design, Email: 1073120824@qq.com.

* Corresponding author: Liuyang Shen, $\mathrm{PhD}$ candidate, research interests: agricultural products processing technology. College of engineering, Northeast Agricultural University, No.600, Changjiang Road, Xiangfang District, Harbin 150030, China. Tel: +86-451-55191021, Email: feiyanghero@163.com. cost and processing time for the extraction of walnut kernels ${ }^{[4]}$. Therefore, the highly efficient methods and devices used to crack walnut shell are urgent to be developed and designed based on the physical characteristics and mechanical properties of walnut, which is an important prerequisite for the design and improvement of a walnut cracking machine ${ }^{[5]}$.

In recent years, many scholars have done many researches to investigate the micro and macro mechanical properties of nuts crops. Generally speaking, compression or shear tests are used to study the macro mechanical properties, and the FEA method is considered as one of most effective means used to analyze the micro mechanical properties ${ }^{[6]}$. Sharifian et al. ${ }^{[7]}$ investigated the mechanical behaviors of walnut by compression tests to determine the effects of moisture content, loading speed, loading position on the rupture force, specific deformation, and absorbed energy. And the quantitative relationships between cracking conditions and mechanical properties of walnut were determined by compression or impact tests ${ }^{[8-11]}$. Furthermore, researches on the macro mechanical properties of other nut crops such as macadamia nut ${ }^{[12]}$, almond nut ${ }^{[13]}$ and hazelnut ${ }^{[14]}$, also have been widely studied by mechanical experiments, and the results indicated that physical characteristics (moisture content, size, shape, shell thickness) and technological parameters (loading force, compression direction, and loading speed) have significant effects on the mechanical properties of nut crops in terms of the rupture force, deformation, and rupture energy. However, with the rapid development of numerical simulation technology, the FEA method has been widely used in the study of agricultural materials, and have obvious advantages in analyzing the complex mechanical behaviors of agricultural products as compared to the conventional experiment 
methods ${ }^{[15]}$. Dintwa et al. ${ }^{[16]}$ and Kim et al. ${ }^{[17]}$ investigated the dynamic collision and mechanical damage mechanism of apples by using FEA method, respectively. Similarly, Zhang et al. ${ }^{[18]}$ analyzed the mechanical properties of Chinese chestnut cracking based on FEA. Sadrnia et al. ${ }^{[19]}$ and Abbaszadeh et al. ${ }^{[20]}$ carried out the analysis of mechanical properties for watermelon by FEA method. By using the FEA method, Li et al. ${ }^{[21]}$ investigated the internal mechanical damage prediction of tomato in depth using multi-scale finite element models to examine the mechanical behaviors under the compression load, and Liu et al. ${ }^{[22]}$ conducted the numerical simulation analysis of mechanical properties for castor capsule during husking. Meanwhile, Tu et al. ${ }^{[23]}$ revealed the mechanical properties and distribution laws of stress and strain for walnut shell under different conditions by FEA method. In summary, so far investigating the mechanical properties and the cracking characteristics of walnut are mainly performed by the mechanical tests and FEA method. These research work clearly indicate that the mechanical properties of walnut cracking can be analyzed by combining the mechanical tests and FEA method.

The above rich researches on mechanical behaviors of nut crops are mainly carried out by the conventional compression tests and the FEA method. Nevertheless, both of them all only consider the contact form between nuts and cracking parts as a single point, which is not conducive to the propagation of shell cracks and easily cause a lot of broken nut kernels to appear due to the effects of stress concentration. Moreover, the related technical information and data in the scientific literature with regard to the mechanical behaviors of walnuts under different contact forms between walnut and cracking parts are insufficient. Consequently, it is extremely essential to explore the mechanical properties of walnut under different contact forms achieved by the different structural shapes of cracking parts.

The objectives of this study were to examine the effects of different contact forms between walnut and cracking parts on the mechanical behaviors and extraction quality of walnut kernel by combining the tests and the FEA method. The specific aims were to 1) evaluate the macro mechanical properties of walnut and the extraction quality of walnut kernel under different cracking parts based on the compression tests by universal testing machine, 2) investigate the laws of micro mechanical properties by utilizing FEA method to simulate the compression process of walnut under different cracking parts and further to predict mechanical damage of walnut. This work may provide guidance for the design and optimization of key components of cracking machines for walnut or other nut crops.

\section{Materials and methods}

\subsection{Experimental materials}

The walnut samples were obtained from the Aral Regiment No.9, Xinjiang Production and Construction Corps. Wen-185 walnut used in this research is one of the most common variety and widely planted in South Xinjiang, and was selected as the experimental materials. The samples were cleaned to remove the impurities, damaged, and broken ones. And the mechanical tests were done on walnut at moisture contents of $4 \%-8 \%$ wet basis within eight hours after samples were purchased. In November 2016, the compression tests of walnut were carried out in the Key Laboratory of Colleges \& Universities under the Department of Education of Xinjiang Uygur Autonomous Region, which was located in Tarim University, Alar, Xinjiang, China.

\subsection{Determination of geometric parameters of walnut}

Based on the suture line, the Wen-185 walnut was divided into three orientations: vertical orientation (along the vertical direction of suture line), sutural orientation (along the short axis of suture line), longitudinal orientation (along the long axis of suture line), as shown in Figure 1. The weight and size of walnut were measured by electronic balance (FA1104, weighing range of 0-200 g, Anting Electronic Instrument Factory, Shanghai, China) and digital caliper (measuring range of $0-150 \mathrm{~mm}$, resolution of $0.01 \mathrm{~mm}$, error $\pm 0.03 \mathrm{~mm}$ ). When compression experiments of walnut were completed, the shell thickness of walnut at different positions (top, middle and bottom) were measured by using the digital caliper, as shown in Figure 1, and the mean value of shell thickness was $1.53 \mathrm{~mm}$. In addition, the sphericity coefficient of walnut were calculated by using the Equation (1) $)^{[24]}$, and the mean value of $S_{p}$ was 0.91 .

$$
S_{p}=\frac{\sqrt[3]{d_{1} d_{2} d_{3}}}{d}
$$

where, $S_{p}$ is the sphericity coefficient of walnut; $d_{1}$ is the dimension of walnut along vertical orientation, $\mathrm{mm} ; d_{2}$ is the dimension of walnut along sutural orientation, $\mathrm{mm} ; d_{3}$ is the dimension of walnut along longitudinal orientation, $\mathrm{mm} ; d=\operatorname{Max}\left\{d_{1}, d_{2}, d_{3}\right\}, \mathrm{mm}$.
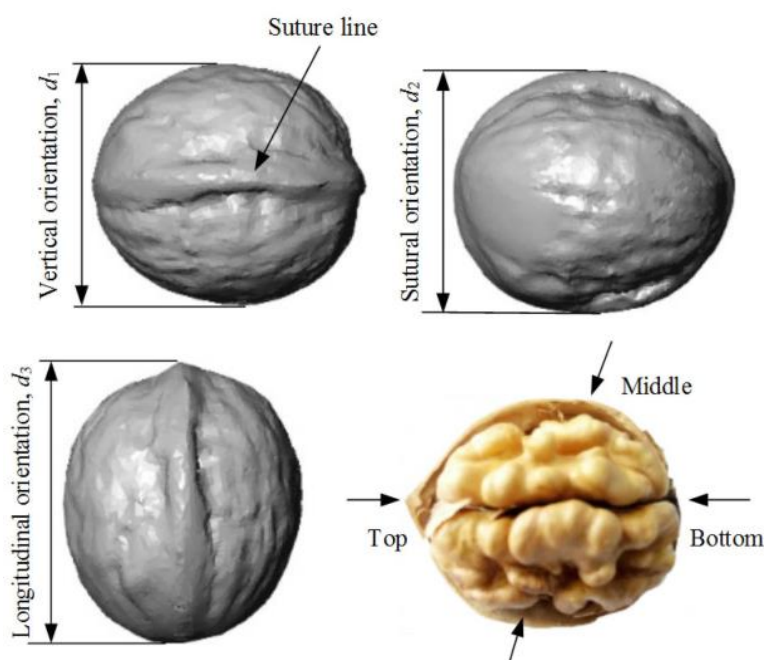

Figure 1 Schematic diagram of measurement for the dimension and the shell thickness of walnut

\subsection{Compression experiments of walnut}

The cracking effects and the mechanical behaviors of walnut under different cracking parts were investigated by performing the compression tests based on the electronic universal testing machine (WD-D3, Zhuoji Testing Intruments Co., Shanghai, China) with microprocessor control, and the main technical parameters of testing machine were as follow: effective force measurement range of 10-5000 N, adjustable loading speed range of 0.001-500 mm/min and accuracy class of 0.5 . The test data of force and displacement during the whole experiments were automatically collected every $0.02 \mathrm{~s}$ by the microprocessor. According to the previous experiments and research results of literature ${ }^{[25,26]}$, a better extraction quality of Wen-185 walnut kernel was achieved when the test control parameters were as follow: loading speed of 300 $\mathrm{mm} / \mathrm{min}$, from the longitudinal orientation to load, and preloading force of $50 \mathrm{~N}$, respectively.

Therefore, the above test control parameters were used in the compression tests of walnut. The whole compression tests of walnut totally included three rounds of duplicate tests. In each 
round of compression test, 60 walnut samples were selected from the experimental materials for mechanical testing, and they were randomly divided into three groups, which were corresponded to three kind of cracking parts, respectively, as shown in Figure 2. Moreover, the mean value of geometric diameter of walnut along the vertical, sutural and longitudinal orientation were $36.76 \mathrm{~mm}$, $35.76 \mathrm{~mm}$ and $40.55 \mathrm{~mm}$, respectively, and average weight was $14.34 \mathrm{~g}$. The structural shapes of cracking parts were flat, conical, and spherical, respectively, and the GB/T 4458.4-2003 of China was used for the dimension marking (the default unit is in $\mathrm{mm}$ ), as shown in Figure 3. In order to prevent walnut slipping and keep the loading force from the longitudinal direction to load in the process of compression tests, the nut with a diameter of $32 \mathrm{~mm}$ was used to fix the position of walnut samples.

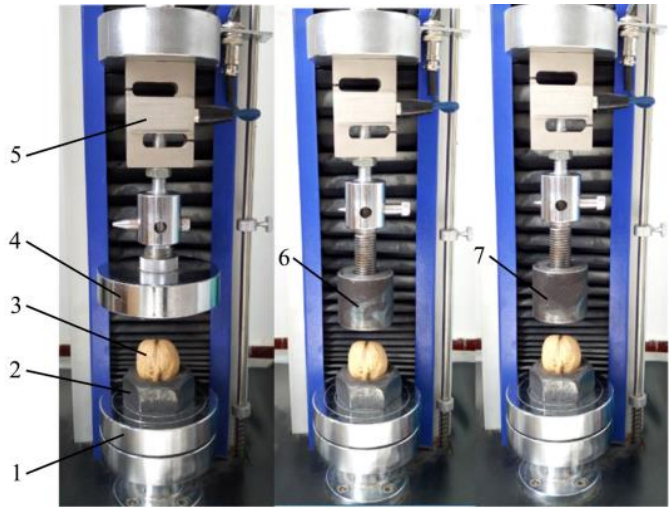

1.Fixed plate 2. Nut 3. Walnut 4. Flat hammerhead 5. Sensor 6. Conical hammerhead 7. Spherical hammerhead

Figure 2 Schematic diagram of compression tests of walnut

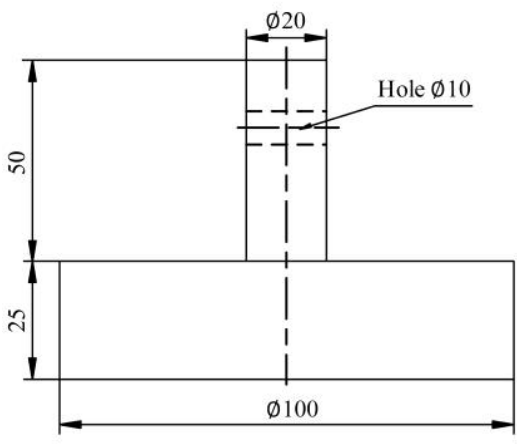

Flate hammerhead

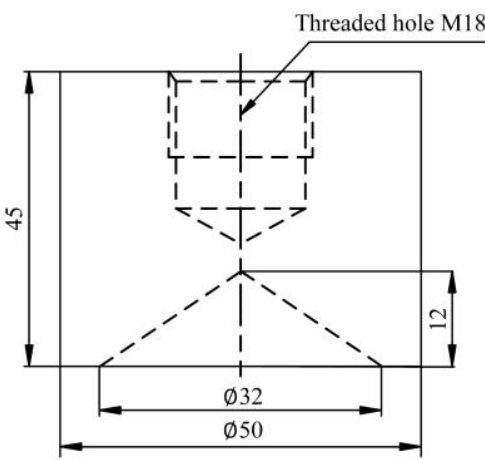

Conical hammerhead

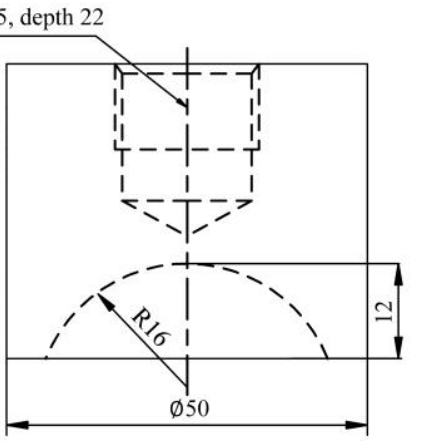

Spherical hammerhead

Figure 3 Schematic diagram of cracking parts

To evaluate the cracking effects of walnut under different cracking parts, the shell-breaking (shell was broken, and kernel was partially or completely exposed) rate, whole kernel ${ }^{[27]}$ (no damaged) rate and first-grade kernel (more than 1/4 whole kernel) rate were used as the evaluation index, as shown in Equation (2):

$$
\left\{\begin{array}{l}
Q_{1}=\frac{N_{1}}{N_{0}} \times 100 \% \\
Q_{2}=\frac{N_{2}}{N_{0}} \times 100 \% \\
Q_{3}=\frac{N_{3}}{N_{0}} \times 100 \%
\end{array}\right.
$$

where, $Q_{1}$ is the shell-breaking rate, $\% ; Q_{2}$ is the first-grade kernel rate, $\% ; Q_{3}$ is the whole kernel rate, $\% ; N_{0}$ is the total number of whole walnut samples; $N_{1}$ is the total number of cracked walnut samples; $N_{2}$ is the total number of first-grade kernels; $N_{3}$ is the total number of whole kernels.

\subsection{FEA of walnut under different cracking parts}

Due to the fact that the compression test results of walnut were not enough to reflect how the internal stress and strain were changing in the compressed walnut, thus the FEA method was used to figure out the complex stress and strain distributions, by which, the causes of cracks for walnut shell could be found out. To begin with, the geometrical model of walnut was built by SolidWorks software (Version 2014, Dassault Systemes S.A, Massachusetts, USA), and the parameters of geometrical model were determined based on statistic results of experimental walnut samples. Then, the FEA model was developed by importing the geometric model into the ANSYS software (Version 15.0, ANSYS Inc., Pittsburgh, Pennsylvania, USA), which was used for analyzing and solving the following FEA model, and the simulation parameters were derived from the related literatures. Moreover, the FEA simulation results were compared with the experimental results, so as to verify the feasibility of FEA results for walnut during compression.

\subsubsection{Establishment of the geometric model of walnut}

Due to a large gap between the shell and the kernel of walnut, and the shell was mainly subjected to the external forces in process of walnut cracking. In order to simplify the analysis model of walnut, therefore, the interactions between kernel and shell were not considered, only to build the three-dimensional (3D) model of walnut shell used for simulation analysis. According to the measured parameters of walnut samples and cracking parts, the $3 \mathrm{D}$ model of walnut shell and cracking parts were established by SolidWorks software, then the model were imported into the ANSYS software to establish the FEA model. The simulation parameters of FEA model were referenced the literatures, and the specific parameters are shown in Table 1.

Table 1 FEA model parameters of walnut

\begin{tabular}{lcc}
\hline \multicolumn{1}{c}{ Parameters name } & Value & References \\
\hline Density/kg $\mathrm{m}^{-3}$ & 470.0 & {$[28]$} \\
Elastic modulus/MPa & 10.0 & {$[28]$} \\
Poisson's ratio & 0.29 & {$[28]$} \\
Tensile yield strength/MPa & 30.0 & {$[29]$} \\
Compressive yield strength/MPa & 3.0 & {$[29]$} \\
\hline
\end{tabular}

\subsubsection{Meshing and boundary conditions}

The walnut shell was treated as the brittle material due to a low moisture content of experimental walnut samples, the failure mode was considered as brittle failure, therefore the failure criterion was used the strength criterion of brittle fracture failure ${ }^{[28]}$. The model of cracking parts was used the Solid186 unit, and shell model was used the Solid187 unit, and CONTAC and TARGE were selected as the contact unit, which was used to calculate the interactions between the shell and cracking parts ${ }^{[23]}$. The target surface (shell) 
was defined by TARGE170 unit, and the contact surface (cracking parts) was defined by CONTAC174 unit. The parameters of shell materials for simulation model was derived from the Table 1, and the material of cracking parts and nut were structural steel selected from default material library of ANSYS software. The method of mesh partition was automatically generated, and the mesh size was $2 \mathrm{~mm}$. And the model of flat compression was divided into 44 583 units and 78148 nodes; the model of conical compression was divided into 36551 units and 65415 nodes; the model of spherical compression was divided into 36837 units and 65950 nodes. The bottom of nut was restrained to be fixed, and the mean value of rupture force of walnut in the compression tests was taken as the applied load of FEA simulation, as shown in Figure 4.
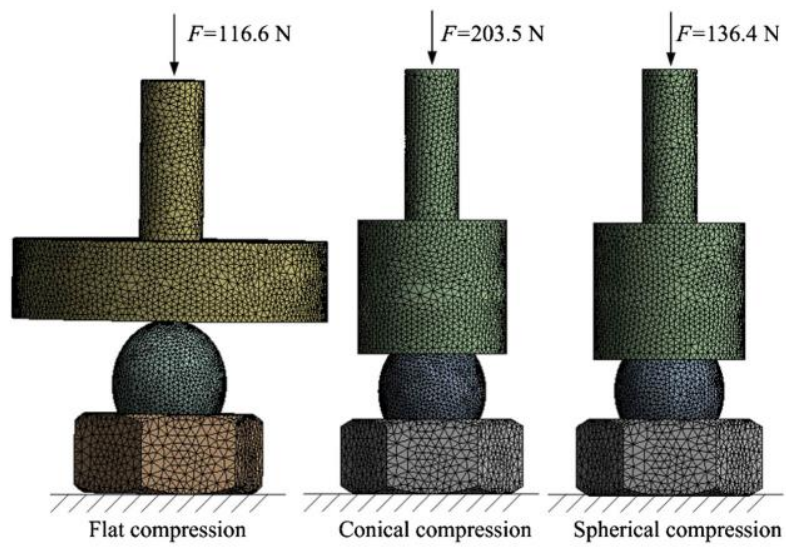

Figure 4 Schematic diagram of meshing and constraint

\subsection{Statistical analysis}

The data were analyzed by one-way analysis of variance (ANOVA) and the Duncan's test method using SPSS statistics software (Version 19.0, SPSS Inc., Chicago, IL, USA). The statistical significance for differences was tested at 5\% probability level $(p<0.05)$. And the Origin software (Version 9.0, OriginLab Corp., Massachusetts, USA) was used for the data graphing.

\section{Theoretical analysis on walnut shell cracking}

\subsection{Critical condition of walnut shell cracking}

According to the theory of elasticity, the walnut shell was deformed and produced instability cracks when the external load increased to a certain value. The critical stress of shell instability was given by the following equation ${ }^{[30]}$ :

$$
P=\frac{2 E}{\sqrt{3\left(1-\mu^{2}\right)}}\left(\frac{h}{r}\right)^{2}
$$

where, $P$ is the critical stress, MPa; $E$ is the modulus of elasticity, $\mathrm{MPa} ; \mu$ is Poisson's ratio; $h$ is the thickness of shell, $\mathrm{mm} ; r$ is the radius of walnut, $\mathrm{mm}$.

As shown in the Equation (3), the critical stress $P$ was proportional to $h$ in terms of some types of walnut with a certain $E$, $\mu$, and $r$, and the shell thickness of walnut depended on its position. Based on the measurement and survey of walnut, the related parameters of walnut were as follow: $E=10 \mathrm{MPa}, \mu=0.29, r=$ $17.32 \mathrm{~mm}$. According to the Equation (3), the critical stress $P$ was calculated based on the thickness of shell at different positions of walnut (Figure 1), and the specific value of critical stress is shown in Table 2 .

\subsection{Critical condition of crack propagation}

The cracks were produced on the shell when a walnut was subjected to a large external load. According to the fracture mechanics, the cracks were divided into three types (I, II, and III), as shown in Figure 5. Type I, II and III were opening cracks, sliding cracks, and tearing cracks, respectively. After the walnut shell was broken, the cracks were mainly type I and type III $^{[30]}$.

Table 2 Critical stress at different positions of walnut

\begin{tabular}{ccc}
\hline Position & Average thickness of shell/mm & Critical stress/MPa \\
\hline Top & 1.54 & 1.073 \\
Middle & 1.52 & 1.059 \\
Bottom & 1.52 & 1.059 \\
\hline
\end{tabular}
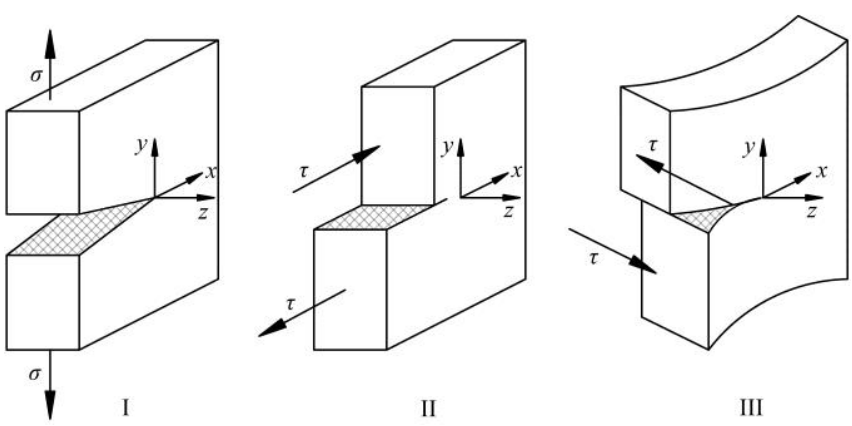

Figure 5 Crack styles

A principle used to describe the variations of rapid crack propagation and the brittle fractures of shell was proposed by Berry ${ }^{[31]}$. Influenced by the external forces, stresses concentrate surrounding the cracks and it propagated until the external force reached a critical value. The relationship between crack length and critical stress was expressed as Equation (4):

$$
\sigma_{c}=\sqrt{\frac{2 E \gamma}{\pi \cdot C}}
$$

where, $\sigma_{c}$ is the critical stress, MPa; $E$ is the elasticity modulus of walnut shell, MPa; $\gamma$ is the surface energy per unit area, $\mathrm{kJ} / \mathrm{m}^{2}$; and $C$ is the length of crack, mm.

Regarded as the properties of wood, the surface energy per unit area of walnut was $0.12 \mathrm{~kJ} / \mathrm{m}^{2}$. The average length of crack after walnut broken was measured to be $16.2 \mathrm{~mm}$. Combined with the above parameters of walnut and the Equation (4), the critical stress $\sigma_{c}$ for crack propagation was $0.217 \mathrm{MPa}$. Therefore, the external load needed to exceed $0.217 \mathrm{MPa}$ and the cracks were further propagated when the shell was broken.

\subsection{Force analysis of walnut under different cracking parts}

The contact forms between walnut and cracking parts (flat hammerhead, conical hammerhead and spherical hammerhead) were corresponding to single point, circle line and spherical surface, respectively. And the schematic diagram of walnut subjected to force under different cracking parts is shown in Figure 6.

In order to analyze the differences of walnut subjected to force under different cracking parts and represent the condition for minimal force, a modified Hertz's contact model developed by Kate et al. ${ }^{[32]}$ was introduced to evaluate it and the model was expressed as Equation (5):

$$
F=\left(\frac{D^{3} S_{p} d}{1.12}\right)^{\frac{1}{2}}\left(\frac{E}{1-\mu^{2}}\right)
$$

where, $F$ is the rupture force of walnut, $\mathrm{N} ; D$ is the compression deformation of walnut, $\mathrm{mm} ; S_{p}$ is the sphericity coefficient of walnut; $d$ is the diameter of walnut, $\mathrm{mm} ; E$ is the elasticity modulus, $\mathrm{MPa}$; and $\mu$ is Poisson's ratio.

For a certain variety and specific size of walnut, the value of $S_{p}$, $d, E$, and $\mu$ were determined, and thus the value of $F$ was positive correlation with $D$ according to Equation (5). Owning to the 
different contact forms between walnut and cracking parts, the compression deformation varied with the contact areas due to the numbers of stress concentration points.
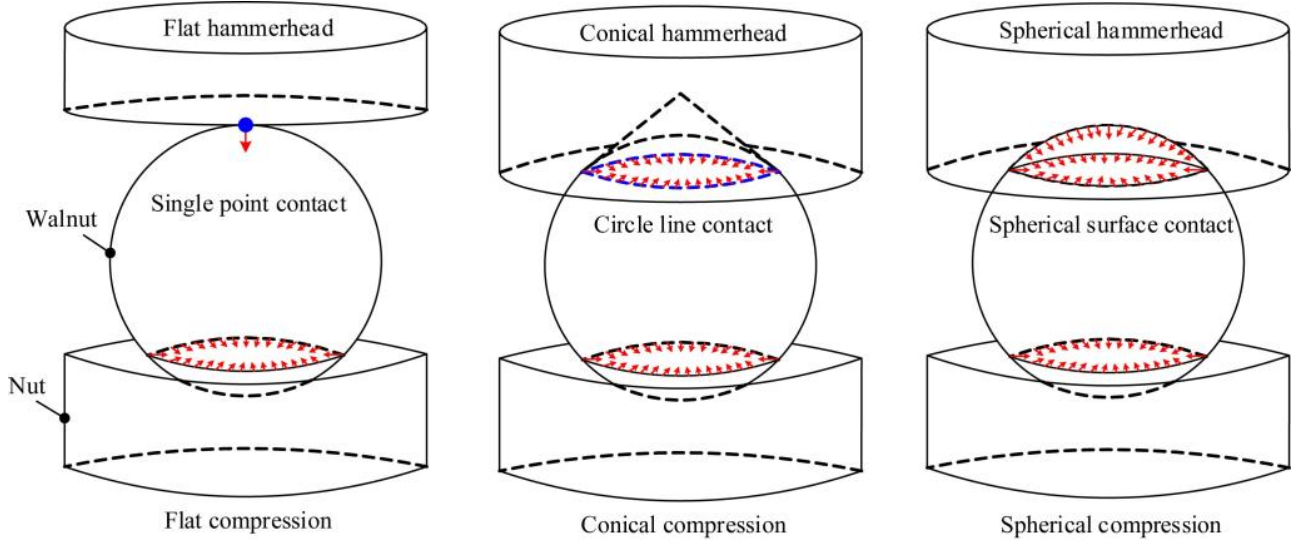

Figure 6 Schematic diagram of walnut subjected to force under different cracking parts

\section{Results and discussion}

\subsection{Analysis of mechanical properties of walnut}

The mechanical properties of walnut under different cracking parts were expressed in terms of the maximum force required to rupture the shell and specific deformation. The mean value of test results for each group corresponding to one kind of cracking parts was taken as the final results, and the corresponding parameters of force and deformation were plotted as the force-deformation curves using Origin software, as shown in Figure 7.

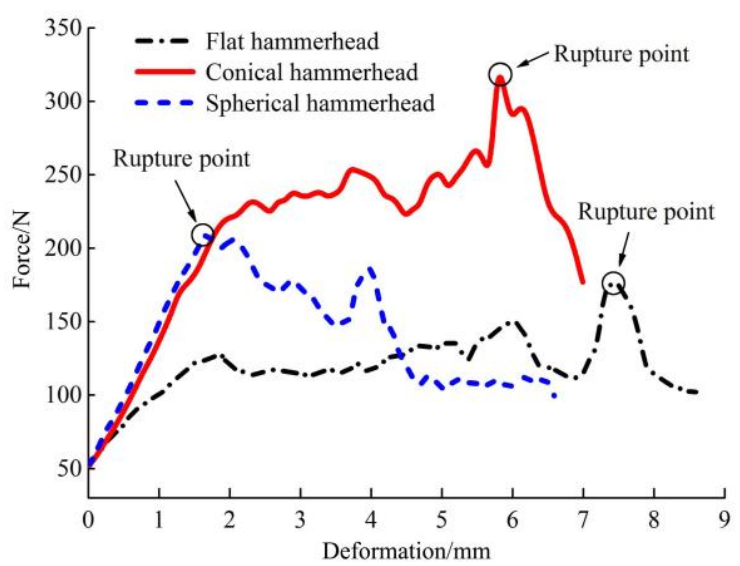

Figure 7 Force-deformation curves of walnut under different cracking parts

As shown in Figure 7, the relationships between force and deformation of walnut were nonlinear curves under three kinds of cracking parts. In initial stage of compression, the relationships between force and deformation of walnut were basically linear, which indicated that elastic deformation of shell mainly occurred in this stage. With the cracking parts move downward, the force and deformation were gradually increased, and the elastic and plastic deformation occurred simultaneously, but the plastic deformation was dominated in this stage. In the later stage of compression, when the value of force was up to the peak (rupture point), the shell was rapidly fractured ${ }^{[33]}$, and obvious cracks occurred at the macro level. With a continuous increase of deformation, however, the reasons for the rupture force having a suddenly sharp fall were attributed to the fact that the structural strength of shell rapidly reduced due to a low anti-compression capacity caused by the generation and propagation of shell cracks.

In addition, the value of force was firstly up to the peak under the spherical compression, with the force of $211.83 \mathrm{~N}$ and the deformation of $1.68 \mathrm{~mm}$, which showed that the time to rupture shell was the shortest and the shell was most easily to be cracked in this way. On the contrary, the value of force reached finally the peak under the conical compression, with the force of $328.55 \mathrm{~N}$ and the deformation of $5.79 \mathrm{~mm}$, which indicated that the time to rupture shell was the longest and the shell was difficult to be cracked. For the flat compression, the corresponding rupture force and deformation were $176.84 \mathrm{~N}$ and $7.49 \mathrm{~mm}$, respectively. Therefore, the method of spherical compression was the best way to achieve walnut cracking.

\subsection{Statistical results and analysis of cracking effects}

According to the Equation (2), the evaluation indexes were calculated for the cracking effects of walnut under the different cracking parts, and the specific statistical results are shown in Figure 8. In terms of the shell-breaking rate, it reached more than $85 \%$ for the three kinds of cracking parts, but little significant differences $(p>0.05)$, which showed that cracking parts had no significant effects on shell-breaking rate. However, for the yield of first-grade kernel, it was up to $88.33 \pm 2.89 \%, 85.00 \pm 5.0 \%$, $70.00 \pm 5.0 \%$ for the spherical, conical and flat hammerhead, respectively, which indicated that cracking parts had significant effects $(p<0.05)$ on the yield of first-grade kernel. Likewise, in terms of the yield of whole kernel, it was $80.00 \% \pm 5.0 \%, 63.33 \% \pm$

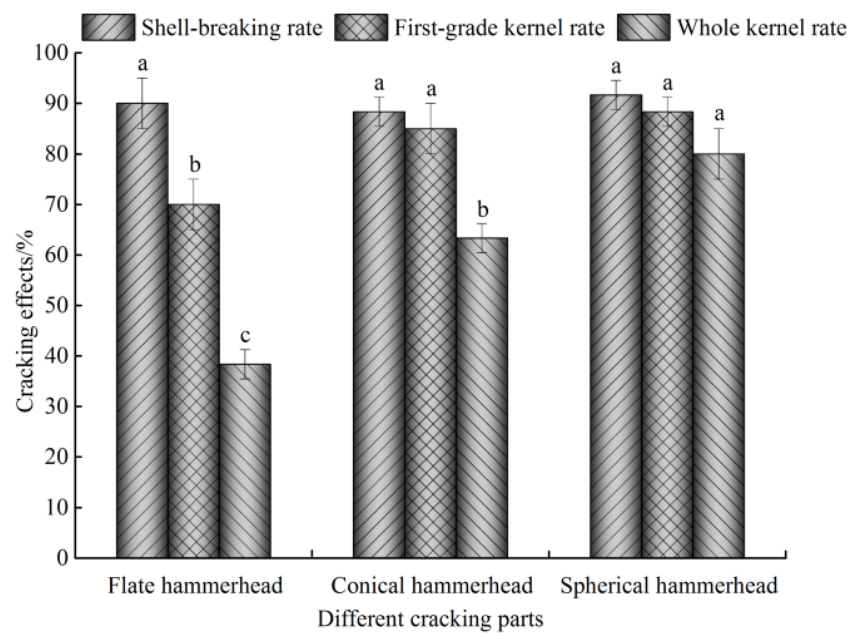

Note: The same letters indicate that there is no significant difference $(p>0.05)$ for the same evaluation index under different cracking parts.

Figure 8 Cracking effects of walnut under different cracking parts 
$2.89 \%, 38.33 \% \pm 2.89 \%$ for the spherical, conical and flat hammerhead, respectively, which indicated that the cracking parts had extremely significant effects $(p<0.01)$ on the yield of whole kernel. Overall, the cracking effects of walnut for spherical hammerhead was the best, and the yield of shell-breaking, first-grade kernel and whole kernel was up to $91.67 \% \pm 2.89 \%$, $88.33 \% \pm 2.89 \%, \quad 80.00 \% \pm 5.00 \%$, respectively. Thus, the structural shape of cracking parts may be designed into the spherical structure to reduce the mechanical damage of walnut kernel during walnut cracking.

\subsection{Analysis of finite element simulation results}

The results of finite element simulation of walnut under different cracking parts were mainly presented by the distribution diagram of equivalent stress and strain, as shown in Figure 9. In addition, the actual crack shapes of walnut under different cracking parts are shown in Figure 10.

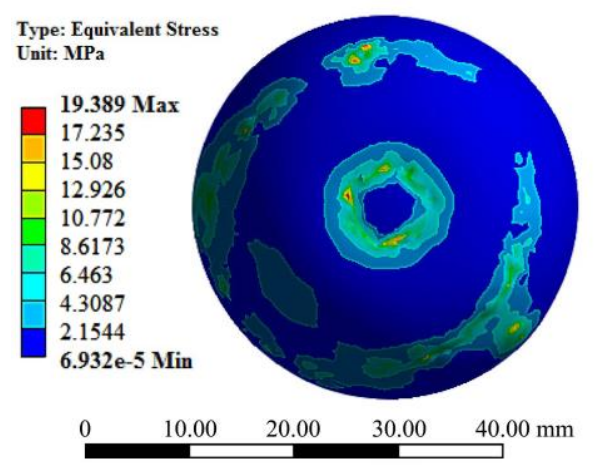

a. Distribution diagram of equivalent stress for walnut under flat compression

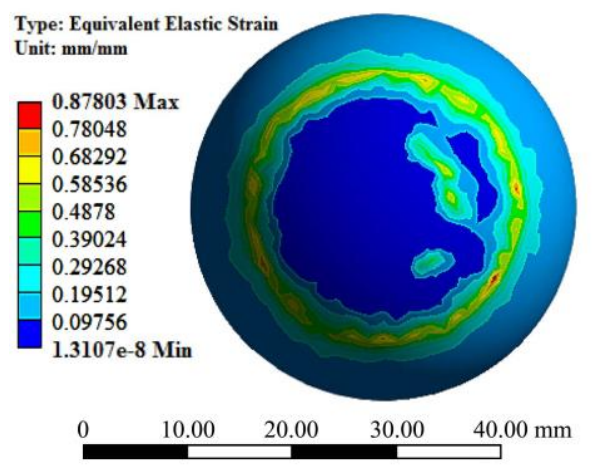

d. Distribution diagram of equivalent strain for walnut under conical compression

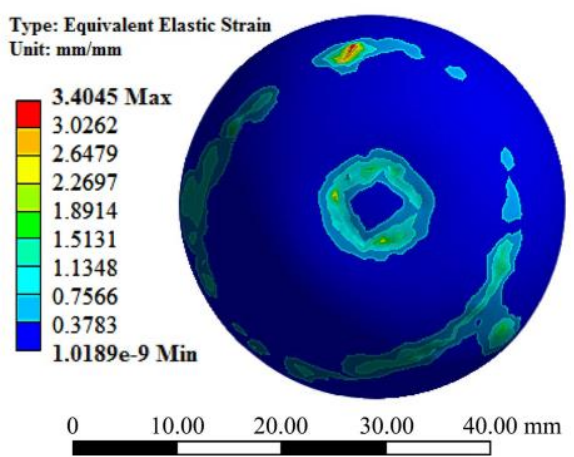

b. Distribution diagram of equivalent strain for walnut under flat compression

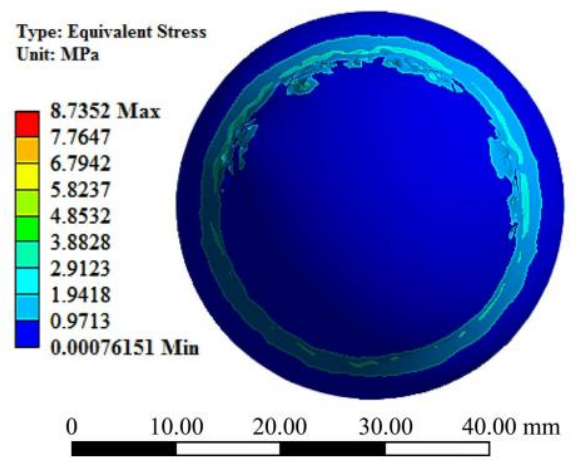

e. Distribution diagram of equivalent stress for walnut under spherical compression

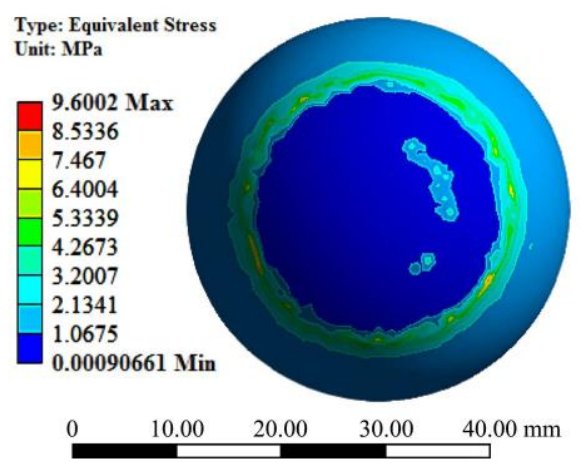

c. Distribution diagram of equivalent stress for walnut under conical compression

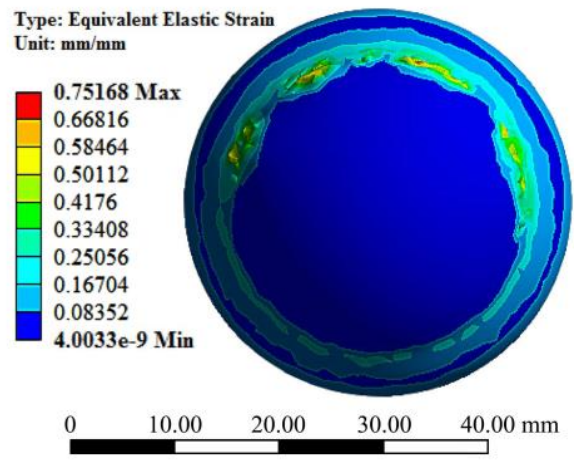

f. Distribution diagram of equivalent strain for walnut under spherical compression

Figure 9 Distribution diagram of finite element simulation for walnut under different cracking parts

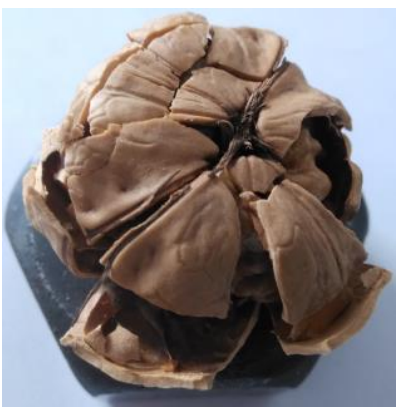

(a) Crack shapes of compressed walnut under flat compression

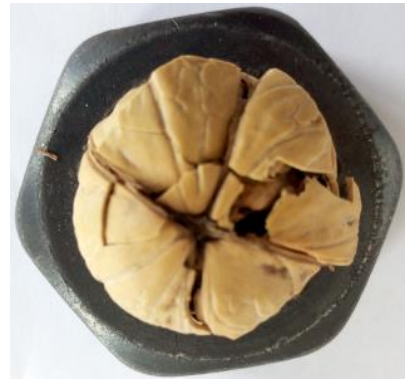

(b) Crack shapes of compressed walnut under conical compression

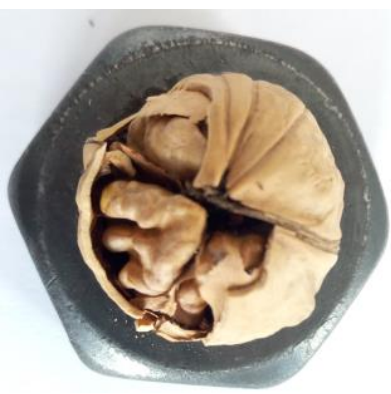

(c) Crack shapes of compressed walnut under spherical compression

Figure 10 Crack shapes of walnut under different cracking parts

It can be seen from the Figure 9, the maximum of equivalent stress and strain under flat hammerhead were $19.389 \mathrm{MPa}$ and 3.4045 , respectively, while the minimum of equivalent stress and strain were $6.932 \times 10^{-5} \mathrm{MPa}$ and $1.0189 \times 10^{-9}$, respectively. Similarly, the maximum of equivalent stress and strain for conical hammerhead were 9.6002 MPa and 0.87803, respectively, while the minimum of equivalent stress and strain were $9.0661 \times 10^{-4} \mathrm{MPa}$ and $1.3107 \times 10^{-8}$, respectively. For the spherical hammerhead, the maximum of equivalent stress and strain were $8.7352 \mathrm{MPa}$ and 0.75168 , respectively, while the minimum of equivalent stress and strain were $7.6151 \times 10^{-4} \mathrm{MPa}$ and $4.0033 \times 10^{-9}$, respectively. Compared with simulation results under three kinds of cracking parts, the rank order of relationships between them was flat > conical > spherical, which was consist with the analysis of compression test results.

As shown in Figures 9a and 9b, the distribution regions of equivalent stress and strain were approximately circular region taken the loading point as center, and the 'pit' was obvious in loading center. Owing to the similar distribution diagram of the equivalent stress and strain, the distribution of stress and strain for walnut had no clear directionality under the action of flat hammerhead. The reasons of shell rupture were mainly due to the point contact form between walnut and flat hammerhead, and walnut shell was subjected to concentrated force, resulting in the 
occurrence of local rupture of shell. It could be predicted that the rupture form was from the loading center to spread around shell, and the local cracks were less generated and much walnut kernels were broken due to the poor ductility of shell, as shown in Figure 10a. Therefore this shape of cracking parts was not conducive to extract kernel in process of walnut cracking.

Similarly, the distribution regions of equivalent stress and strain were approximately annular region, as shown in Figures 9c and 9d. The stress and strain were mainly produced in the contact between walnut and conical part, and the distribution of stress and strain had no clear directionality. The reasons of shell rupture were mainly due to the annular line contact form between walnut and conical hammerhead, and shell was subjected to multi-point force, which induced lots of cracks to be produced. It could be predicted that the rupture form was from the suture line to spread around shell. Due to the fact that the stress at the top of walnut was smaller, the deformation propagated toward the top of shell when the conical hammerhead move downward, and stress and strain were mainly generated in the contact line, so less damage of walnut kernel produced, as shown in Figure 10b, and it was a better way to crack walnut.

Likewise, the distribution regions of equivalent stress and strain were also approximately annular region seen from the Figures 9e and 9f, but the distribution were more inclined to one side, therefore the distribution of stress and strain had a certain directionality. And the stress and strain existed in the contact regions between walnut and spherical parts. The reasons of shell rupture were attributed to spherical surface contact form between walnut and spherical hammerhead, and the middle part of shell was subjected to smaller stress, which induced crack propagation from the contact place to the top of walnut. It could be predicted that the cracks of shell had obvious directionality, and the highest extraction quality of walnut kernel may be achieved under the spherical compression, as shown in Figure 10c, and hence it was a best way to crack walnut.

FEA results of compressed walnuts showed that 1) For a single point contact under the flat compression, large amount of broken kernel appeared because the ruptured area was partial region and cracks were less produced; 2) For a circle line contact under the conical compression, a better cracking effect and extraction quality of walnut kernel could be obtained than the flat compression since more local cracks were produced; 3) For a spherical surface contact under the spherical compression, the integrity of extracting walnut kernel was the best due to the fact that more cracks were generated and the cracks had obvious trend to be propagated. Therefore, the spherical surface contact between walnut and cracking parts should be considered to design into the structure shape of key components for cracking machines, which was consistent with the analysis of compression test results.

\subsection{Comparison between experiments and FEA}

In order to verify the feasibility of FEA results, the test results were compared with the FEA results, and the deformation of walnut was selected as comparison item between compression tests and FEA, as shown in Table 3.

The comparison showed that FEA results were basically close to the experiment results, which indicated that FEA model was feasible to simulate and analyze the mechanical properties of compressed walnut under different cracking parts. There were, however, still some differences between the FEA results and test results, and the maximum difference of deformation was $12.8 \%$. The reasons of differences maybe are diverse and caused by: 1) the error of compression test data; 2) the error of simulation parameters when creating the FEA model; 3) the interactions between walnut kernel and shell are neglected; 4) the geometric size of FEA simulation model is not exactly the same with the actual size of walnut; 5) the mechanical properties of different parts of walnut are different, but it is equally treated in the simulation model; 6) there are some differences between model load and actual load.

Table 3 Comparison between test value and FEA value

\begin{tabular}{|c|c|c|c|c|}
\hline \multirow{2}{*}{$\begin{array}{l}\text { Cracking } \\
\text { parts }\end{array}$} & \multirow{2}{*}{$\begin{array}{l}\text { Loading } \\
\text { force/N }\end{array}$} & \multicolumn{2}{|c|}{ Compare item (deformation)/mm } & \multirow{2}{*}{ Ratio } \\
\hline & & Test values & FEA values & \\
\hline Flat hammerhead & 116.6 & 4.183 & 4.218 & 0.992 \\
\hline Conical hammerhead & 203.5 & 3.304 & 3.534 & 0.935 \\
\hline Spherical hammerhead & 136.4 & 3.105 & 2.753 & 1.128 \\
\hline
\end{tabular}

Note: The loading force is derived from the mean value of force of walnut in compression tests; the test values are derived from the mean value of deformation of walnut in compression tests; the FEA values of deformation are derived from the simulation results.

\section{Conclusions}

The compression experiments and finite element simulation analysis were integrated to investigate the mechanical properties and cracking effects of walnut under different cracking parts. The relationships between force and deformation of walnut were nonlinear in the condition of compression, and its rupture process mainly included three stages (elastic, plastic and elastic-plastic stage). Based on the FEA results, the cracks of walnut shell had obvious trends and easily to be propagated due to the fact that the contact form between walnut shell and spherical hammerhead was approximately spherical surface, which caused the integrity of extracting kernel in the processing of walnut cracking. On the contrary, the rupture effects of walnut shell were partial fracture because the contact form between walnut and flat hammerhead was approximately a single point, which easily caused large amount of broken kernel appeared. The established FEA model of walnut can be used to analyze the mechanical properties of walnut by the comparison between experiment results and FEA results.

The suitable processing way to achieve the walnut cracking with a high extraction quality of walnut kernel was the spherical compression. Therefore, the spherical surface contact between walnut and cracking parts may be considered to design into the structure shape of key components of cracking machines, and the research results may provide references for the structural design and optimization of key components of cracking machines for walnut or other nut crops.

\section{Acknowledgments}

The authors gratefully thank the financial support provided by the National Natural Science Foundation of China (Grant No.31160196, No.31660475) for this research project.

\section{[References]}

[1] Christopoulos M V, Tsantili E. Oil composition in stored walnut cultivars-quality and nutritional value. Eur J Lipid Sci Tech, 2015; 117(3): 338-348.

[2] FAO. Statistical database. http://faostat.fao.org. Accessed to web.

[3] Li Z X, Yang J, Yang L L, Yang Z Q, Liu K, Liu J, et al. The present status and trends of the processing for the featured forest fruits in southern Xinjiang. Xinjiang Agricultural Sciences, 2010; 47(11): 2177-2183. (in Chinese)

[4] Ghafari A, Chegini G R, Khazaei J, Vahdati K. Design, construction and performance evaluation of the walnut cracking. International Journal of 
Nut and Related Science, 2011; 2(1): 11-16.

[5] Cao C M, Sun S, Ding R, Li B, Wang S. Experimental study on mechanical characteristics of nut rupturing under impact loading. Int $\mathbf{J}$ Agric \& Biol Eng, 2017; 10(1): 53-60.

[6] Lu B, Hu C, Wang X U, Tang X Y, Niu R K, Li C F. Compressive mechanical properties test and finite element analysis of winter jujube during crisp ripe period in South Xinjiang, China. IAEJ, 2016; 25(4): 245-256.

[7] Sharifian F, Derafshi M H. Mechanical behavior of walnut under cracking conditions. J Applied Sci, 2008; 8(5): 886-890.

[8] Gharibzahedi S M T, Mousavi S M, Hamedi M, Khodaiyan F, Dadashpour A. Mechanical behavior of Persian walnut and its kernel under compression loading: an experimental and computational study. J Food Process Preserv, 2012; 36(5): 423-430

[9] Altuntas, E. The effects of moisture content, compression speeds, and axes on mechanical properties of walnut cultivars. Food Bioprocess Technol, 2011; 4(7): 1288-1295.

[10] Shahbazi F. Effective conditions for extracting higher quality kernels from walnuts. Qual Assur Saf Crop, 2013; 5(3): 199-206.

[11] Shahbazi F. Effects of moisture content and impact energy on the cracking characteristics of walnuts. Int J Food Eng, 2014; 10(1): 165-181.

[12] Braga G C, Couto S M, Hara T, Neto J T P A. Mechanical behaviour of macadamia nut under compression loading. J Agric Eng Res, 1999; 72(3): 239-245.

[13] Aktas T, Polat R, Atay U. Comparison of mechanical properties of some selected almond cultivars with hard and soft shell under compression loading. J Food Process Eng, 2010; 30(6): 773-789.

[14] Güner M, Dursun E, Dursun İ G. Mechanical behaviour of hazelnut under compression loading. Biosyst Eng, 2003; 85(4): 485-491.

[15] Liu Z, Scanlon M G. Modelling indentation of bread crumb by finite element analysis. Biosyst Eng, 2003; 85(4): 477-484.

[16] Dintwa E, Zeebroeck M V, Ramon H, Tijskens E. Finite element analysis of the dynamic collision of apple fruit. Postharvest Biol Tec, 2008; 49(2): 260-276.

[17] Kim G W, Do G S, Bae Y, Sagara Y. Analysis of mechanical properties of whole apple using finite element method based on three-dimensional real geometry. Food Sci Technol Res, 2009; 14(4): 329-336.

[18] Zhang R R, Li X Y, Wang W, Zhang J, Liu J. Analysis of mechanical properties of Chinese chestnut cracking based on the FEM. Transactions of the CSAE, 2008; 24(9): 84-88. (in Chinese)

[19] Sadrnia H, Rajabipour A, Jafari A, Javadi A, Mostofi Y, Kafashan J, Dintwa E, Baerdemaeker J D. Internal bruising prediction in watermelon compression using nonlinear models. J Food Eng, 2008; 86(2): 272-280.

[20] Abbaszadeh R, Rajabipour A, Sadrnia H, Mahjoob M J, Delshad M, Ahmadi H. Application of modal analysis to the watermelon through finite element modeling for use in ripeness assessment. J Food Eng, 2014 127(4): 80-84

[21] Li Z G, Li P P, Yang H L, Liu J Z. Internal mechanical damage prediction in tomato compression using multiscale finite element models. J Food Eng, 2013; 116(3): 639-647.

[22] Liu R K, Cheng X X, Xiao Z H, Li C Z, Huang Z H, Ye H Q. Finite element simulation on mechanical property of castor capsule during husking. Journal of the Chinese Cereals and Oils Association, 2015; 30(5): 62-66. (in Chinese)

[23] Tu C, Yang W, Yin Q J, Lv J L. Optimization of technical parameters of breaking macadamia nut shell and finite element analysis of compression characteristics. Transactions of the CSAE, 2015; 31(16): 272-277. (in Chinese)

[24] Shahbazi F. Effects of moisture content and impact energy on the cracking characteristics of walnuts. Int J Food Eng, 2014; 10(1): 165-181.

[25] Li Y, Zhang $\mathrm{H}$, Hao Z C. Experiment on shell-breaking and kernel-fetching for Wen-185 walnut. Journal of Agricultural Mechanization Research, 2014; (7): 174-177. (in Chinese)

[26] Zhang H, Ma Y, Lan H P, Li Y, Zhang R L, Zhang R. Study on test of cracking walnut and fetching kernel of Xinjiang Wen 185 . Journal of Anhui Agricultural Science, 2014; 42(21): 7187-7190. (in Chinese)

[27] Nahal A M, Arabhosseini A, Kianmehr M H. Separation of shelled walnut particles using pneumatic method. Int J Agric \& Biol Eng, 2013; 6(3): 88-93.

[28] Shi J X, Zhao H J, Xin D J. Technology for breaking walnut shell based on finite element analysis. Transactions of the CSAE, 2005; 21(3): 185-188. (in Chinese)

[29] Zhou J, Shi J X. Finite element mechanics analysis for breaking walnut shell in gas explosion type. Journal of Agricultural Mechanization Research, 2014; (12): 65-69. (in Chinese)

[30] Liu M Z, Li C H, Zhang Y B, Jia D Z, Yang M, Hou Y L. Semi-theoretical analyses on mechanical performance of flexible-belt shearing extrusion walnut shell crushing. Appl Eng Agric, 2016; 32(4): 459-467.

[31] Berry J P. Some kinetic considerations of the Griffith criterion for fracture I: equations of motion at constant force. J Mech Phys Solids, 1960; 8(3): 194-206.

[32] Kate A E, Sarkar A, Shahi N C, Lohani U C. Cracking force analysis for apricot pit decortication based on mathematical model of Hertz's theory. Int J Food Prop, 2015; 18(11): 2528-2538.

[33] Liu M Z, Li C H, Zhang Y B, Yang M, Hou Y L, Gao L X. Shell crushing mechanism analysis and performance test of flexible-belt shearing extrusion for walnut. Transactions of the CSAM, 2016; 47(7): 266-273. (in Chinese) 